\title{
Topological phase transition and thermal Hall effect in kagome ferromagnets
}

\author{
Fengjun Zhuo (卓冯骏), ${ }^{1, *}$ Hang $\mathrm{Li}^{2},{ }^{2}$ and Aurélien Manchon ${ }^{3, \dagger}$ \\ ${ }^{1}$ King Abdullah University of Science and Technology (KAUST), \\ Physical Science and Engineering Division (PSE), Thuwal 23955-6900, Saudi Arabia \\ ${ }^{2}$ School of Physics and Electronics, Henan University, Kaifeng 475004, China \\ ${ }^{3}$ Aix Marseille Univ, CNRS, CINAM, Marseille, France
}

\begin{abstract}
We investigate a new mechanism for phase transitions between topological magnon phases on the breathing kagome lattice ferromagnets with Dzyaloshinskii-Moriya interaction, which is induced by the lattice deformation. With the linear spin-wave theory, we calculate the magnonic Chern number, topological phase diagram and magnon thermal Hall conductivity at low temperature with tunable modulated exchange interactions due to the lattice deformation. We show that the modulated exchange interactions and Dzyaloshinskii-Moriya interaction strength determine the band topology of magnons. We find a sign reversal of the thermal Hall conductivity during topological phase transitions explained in terms of topological edge modes and their propagation directions. We also discuss candidate materials to realize our ideas.
\end{abstract}

\section{INTRODUCTION}

In condensed matter physics, the theoretical proposal and experimental discovery of the topological insulators in electronic systems are the subject of extensive interest in the last decade [1-5]. Physically, the essential concept in topological band theory is the topological invariants linked to the topological protected edge states, when the spin-orbit coupling (SOC) is present and opens a topological nontrivial gap [6, 7]. As the band topology is independent of the statistical regularity of (quasi)particles, such ideas can be realized in bosonic systems such as photons [8-12], phonons [13-15], and magnons [16-21]. Different from electronic charge particles, magnons are electrically neutral bosonic quasiparticles, thus they can propagate over long distances without experiencing a Lorentz force and incuring Joule heating in magnetic insulators. Consequently, magnonic devices offer better prospects to realize next generation low-dissipation memory devices [22-24].

A thermal version of the Hall effect-magnon thermal Hall effect (THE) induced by a temperature gradient has been experimentally observed in insulating pyrochlore ferromagnets [17, 25] and kagome ferromagnets [26, 27]. The finite THE is brought about by an effective SOC derived from the Dzyaloshinskii-Moriya interaction (DMI) [28-30], which tends to cant neighboring spins away from each other and induces nonzero Berry curvatures in momentum space as an effective Lorentz force [16, 31]. Similarly, the band topology and the THE are also predicted theoretically in an insulating ferromagnet with the dipolar interaction [18, 32], honeycomb ferromagnets [33, 34], triangular lattice antiferromagnets [35], noncollinear or noncoplanar kagome antiferromagnets [36-39] and skyrmion crystals [40-42]. In addition, when the topological system has two or more distinct topological phases, a topological phase transition may arise with a gap alternating between closing and reopening [43, 44]. Heretofore topological phase transitions among magnonic topological phase have been reported with the variation of magnetic field [35-38, 45], magnon-phonon coupling [46-48],

\footnotetext{
* fengjun.zhuo@kaust.edu.sa

† manchon@cinam.univ-mrs.fr
}

magnon-magnon interaction [49] and variation of nearestneighboring $(\mathrm{NN})$ or next-nearest-neighbor (NNN) exchange interactions [50-53].

Recent theoretical and experimental quantum matter research on a vanadium-based material $\left[\mathrm{NH}_{4}\right]_{2}\left[\mathrm{C}_{7} \mathrm{H}_{1} 4 \mathrm{~N}\right]\left[\mathrm{V}_{7} \mathrm{O}_{6} \mathrm{~F}_{18}\right]$, or diammonium quinuclidinium vanadium(III,IV) oxyfluoride (DQVOF), as well as the materials $\mathrm{Li}_{2} \mathrm{In}_{1-x} \mathrm{Sc}_{x} \mathrm{Mo}_{3} \mathrm{O}_{8}$ have opened an alternative route for the study of quantum spin liquids on the breathing kagome lattice in the absence of inversion symmetry [54-60]. Moreover, topological properties, especially on higher-order topological insulators, have been widely investigated on the breathing kagome lattice in electronics [61-64], photonics $[65,66]$, acoustic metamaterials [67, 68], electric circuits [69-71], magnetic solitons [72] and mechanical metamaterials [73]. More interestingly, a skyrmion crystal phase with large topological Hall effect was also experimentally observed in $\mathrm{Gd}_{3} \mathrm{Ru}_{4} \mathrm{Al}_{12}$ with a Gd-based breathing kagome lattice [74]. Meanwhile, the topological transport of magnons in breathing kagome lattice ferromagnets has recently attracted increasing interests. Magnonic flat bands coexisting with Dirac nodes at the $\Gamma$ point have been demonstrated when the system holds particle-hole symmetry [75] and first and second order topological magnon insulator (TMI) phases have been identified [76]. However, the topological properties of magnons in breathing kagome lattice ferromagnets remain largely unaddressed.

In this paper, we study the magnon band topology and the topological magnon THE in a breathing kagome ferromagnet with DMI as illustrated in Fig. 1. The unit cell is formed by three spin sublattices A, B and C represented by hollow circles in the green dashed diamond. A nonuniform strain field results a lattice deformation $\delta$ such that three sublattices further away (positive perturbation $\delta>0$ ) or get closer (negative perturbation $\delta<0$ ) from their shared corner of the honeycomb lattice as shown in Fig. 1(b). By increasing the strength of $\delta$ in a breathing kagome ferromagnet, we find a topological phase transition between a TMI with coupled nontrivial edge modes and a trivial magnon insulator with trivial edge modes. The phase transition is accompanied by the evolution of magnon bands that the nontrivial band gap closes and reopens. Furthermore, the evolution of topological phases can 
be experimentally detected by the change of the thermal Hall conductivity. We also demonstrate sign changes of the thermal Hall conductivity with respect to the lattice deformation, which can be explained in terms of the correspondence between the thermal Hall conductivity and the propagation direction of the nontrivial edge modes. Different from Refs. $[75,76]$, the present work investigates how lattice deformation due to nonuniform strain fields influences the band topology and associated thermal transport of magnons via the modification of NN ferromagnetic interactions and DMI [see Eq. (2)]. We also show how the magnonic thermal Hall conductivity reflects the topological phase transitions.

The paper is organized as follows. In Sec. II we introduce our theoretical model and method. Results are presented in Sec. III including the topological phase diagram, topological magnon band tructures, magnonic edge modes, the thermal Hall conductivity, and material consideration. Finally, we conclude with Sec. IV.

\section{MODEL AND METHOD}

\section{A. Spin model Hamiltonian for magnons}

In this paper, we consider the following anisotropic spin model on a 2D breathing kagome lattice, whose Hamiltonian reads

$$
\begin{aligned}
\mathcal{H}= & -\sum_{\langle i j\rangle} J_{i j} \boldsymbol{S}_{i} \cdot \boldsymbol{S}_{j}-J_{2} \sum_{\langle\langle i j\rangle\rangle} \boldsymbol{S}_{i} \cdot \boldsymbol{S}_{j} \\
& +\sum_{\langle i j\rangle} \boldsymbol{D}_{i j} \cdot\left(\boldsymbol{S}_{i} \times \boldsymbol{S}_{j}\right),
\end{aligned}
$$

where $\boldsymbol{S}_{i}$ is the vector of spin operators at site $i$. The first two terms represent the $\mathrm{NN}$ and $\mathrm{NNN}$ ferromagnetic couplings $\left(J_{i j}, J_{2}>0\right)$, respectively. The third term is the out-of-plane NN DMI, where $\boldsymbol{D}_{i j}$ is the DMI vector whose orientation depends on the chirality of the triangles in the kagome lattice. In the breathing kagome lattice, the values of the exchange interactions are modulated by the small displacements of the spins. Following the same methodology as in Refs. [77, 78], we expand the intracell/intercell NN ferromagnetic coupling and DMI (blue/red lines shown in Fig. 1) around its equilibrium value $J_{1}$ and $D$ to linear order

$$
\begin{aligned}
& J_{ \pm}=(1 \mp \sqrt{3} \eta \delta) J_{1}, \\
& D_{ \pm}=(1 \mp \sqrt{3} \eta \delta) D,
\end{aligned}
$$

where subscript $+(-)$ indicates intracell (intercell) NN interactions, and $\eta$ is a parameter that describes the response of the couplings to the displacements of sublattices. Here, we neglect the modulation of the NNN ferromagnetic couplings since they are the higher-order terms. However, this simultaneous changes of NN ferromagnetic interaction and DMI will preserve the collinear ferromagnetic ground state, as discussed in Appendix A.
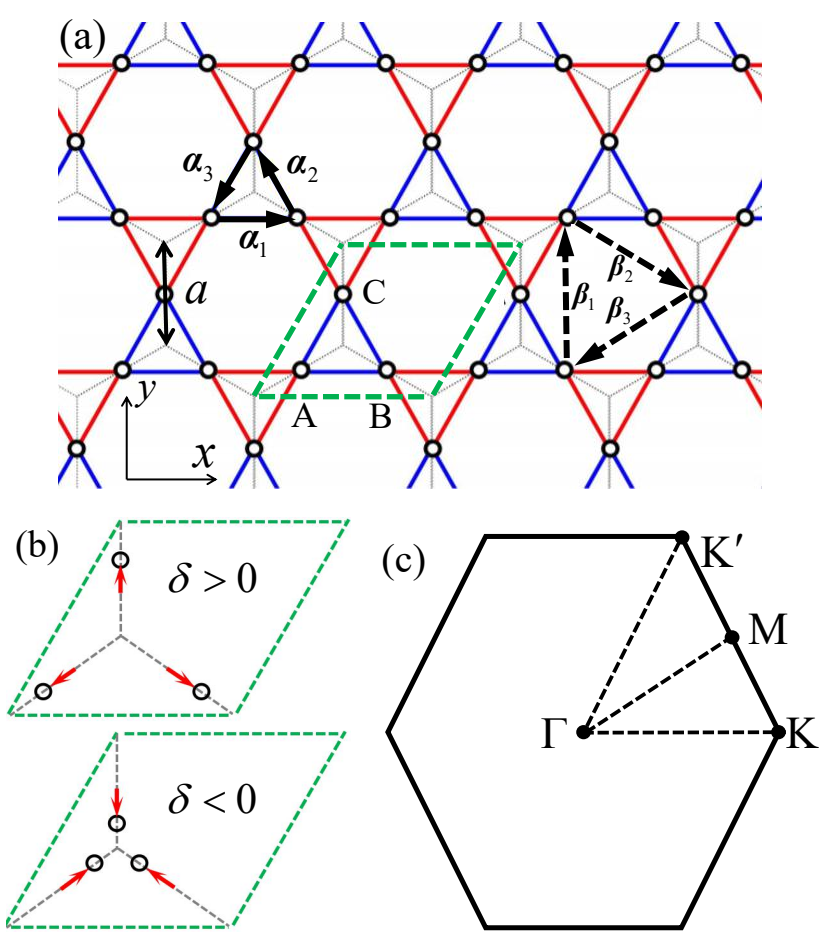

Figure 1. Schematics for the breathing kagome ferromagnet. (a) Unstrained case with $\delta=0$. The gray dashed line is a guide to show the hexagonal symmetry. The blue/red solid lines represent the intracell/intercell NN bonds. $a$ represents the lattice constant. (b) Straining induced perturbations are considered by putting the sublattice postion further away $(\delta>0)$ or closer $(\delta<0)$ to each other, represented by the red arrows. (c) The first Brillouin zone of the reciprocal lattice.

We now turn to a linear spin-wave theory, within which we obtain the tight-binding magnon Hamiltonian in the momentum space. Starting from the spin model Hamiltonian Eq. (1), we express the spin operators in terms of magnon creation operator $\hat{b}_{i}^{\dagger}$ and annihilation operator $\hat{b}_{i}$ by applying the Holstein-Primakoff (HP) transformations [79]

$$
S_{i}^{+} \simeq \sqrt{2 S} b_{i}, \quad S_{i}^{+} \simeq \sqrt{2 S} b_{i}^{\dagger}, \quad S_{i}^{z}=S-b_{i}^{\dagger} b_{i},
$$

where the magnon ladder operators are $S_{i}^{ \pm}=S_{i}^{x} \pm i S_{i}^{y}$. Here, we neglect the magnon-magnon interactions in the low temperature limit, $2 S \gg\left\langle b_{i}^{\dagger} b_{i}\right\rangle$, which gives the higher-order terms in the magnon operators. After a Fourier transformation, we obtain the bilinear magnon Hamiltonian in the momentum space

$$
\mathcal{H}=S \sum_{\boldsymbol{k}} \Psi_{\boldsymbol{k}}^{\dagger} H_{m}(\boldsymbol{k}) \Psi_{\boldsymbol{k}}
$$

with the tight-binding magnon Hamiltonian in a matrix form $H_{m}(\boldsymbol{k})=H_{0}+H_{J}^{\mathrm{NN}}+H_{J}^{\mathrm{NNN}}+H_{D}$ and the magnon operator basis $\Psi_{\boldsymbol{k}}^{\dagger}=\left(b_{A}^{\dagger}, b_{B}^{\dagger}, b_{C}^{\dagger}\right)$. The submatrices of the 


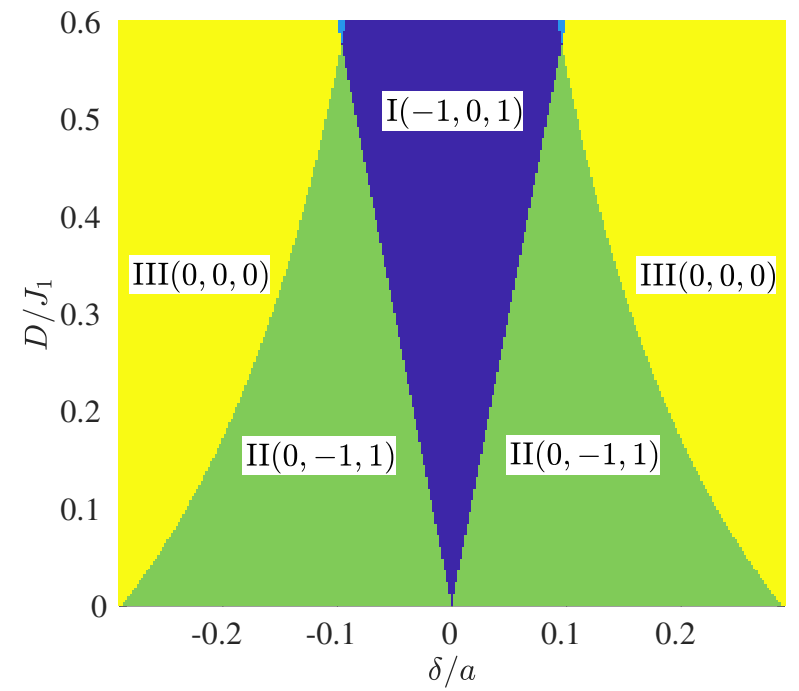

Figure 2. Topological phase diagram of the breathing kagome lattice. Each topological phase is characterized by sets of Chern numbers $\left(C_{1}, C_{2}, C_{3}\right)$.

magnon Hamilton matrix read

$$
\begin{aligned}
H_{0} & =4\left(J_{1}+J_{2}\right) I_{3 \times 3}, \\
H_{J}^{\mathrm{NN}} & =-\left(\begin{array}{ccc}
0 & \gamma_{1} & \gamma_{3}^{*} \\
\gamma_{1}^{*} & 0 & \gamma_{2} \\
\gamma_{3} & \gamma_{2}^{*} & 0
\end{array}\right), \\
H_{D} & =-i\left(\begin{array}{ccc}
0 & d_{1} & -d_{3}^{*} \\
-d_{1}^{*} & 0 & d_{2} \\
d_{3} & -d_{2}^{*} & 0
\end{array}\right), \\
H_{J}^{\mathrm{NNN}} & =-\left(\begin{array}{ccc}
0 & \mu_{1} & \mu_{2} \\
\mu_{1}^{*} & 0 & \mu_{3} \\
\mu_{2}^{*} & \mu_{3}^{*} & 0
\end{array}\right)
\end{aligned}
$$

where $I_{3 \times 3}$ is the $3 \times 3$ identity matrix. The elements of the above matrices read: $\gamma_{i} \equiv J_{+} e^{i \boldsymbol{k} \cdot(1+\delta) \boldsymbol{\alpha}_{i}}+J_{-} e^{-i \boldsymbol{k} \cdot(1-\delta) \boldsymbol{\alpha}_{i}}$ and $d_{i} \equiv D_{+} e^{i \boldsymbol{k} \cdot(1+\delta) \boldsymbol{\alpha}_{i}}+D_{-} e^{-i \boldsymbol{k} \cdot(1-\delta) \boldsymbol{\alpha}_{i}}$ with $\boldsymbol{\alpha}=$ $\left\{\left(\frac{\sqrt{3}}{2}, 0\right),\left(-\frac{\sqrt{3}}{4}, \frac{3}{4}\right),\left(-\frac{\sqrt{3}}{4},-\frac{3}{4}\right)\right\} a$ being the three unstrained NN vectors, $\mu_{i} \equiv J_{2} \cos \left(\boldsymbol{k} \cdot \boldsymbol{\beta}_{i}\right)$ with $\boldsymbol{\beta}=$ $\left\{\left(0, \frac{3}{2}\right),\left(\frac{3 \sqrt{3}}{4},-\frac{3}{4}\right),\left(-\frac{3 \sqrt{3}}{4},-\frac{3}{4}\right)\right\} a$ being the three unstrained NNN vectors as shown in Fig. 1(a).

\section{B. Chern numbers and thermal Hall conductivity}

The particular configuration of the DMI vectors induces textured fluxes in a kagome lattice, which lead to a geometrical phase of the magnon Bloch state [16]. Thus, a nonzero Berry curvature arises and reads

$\Omega_{\lambda \boldsymbol{k}}^{z}=-2 \sum_{\lambda^{\prime} \neq \lambda} \operatorname{Im} \frac{\left\langle\psi_{\lambda \boldsymbol{k}}\left|\partial_{k_{x}} H_{m}\right| \psi_{\lambda^{\prime} \boldsymbol{k}}\right\rangle\left\langle\psi_{\lambda^{\prime} \boldsymbol{k}}\left|\partial_{k_{y}} H_{m}\right| \psi_{\lambda \boldsymbol{k}}\right\rangle}{\left(\varepsilon_{\lambda \boldsymbol{k}}-\varepsilon_{\lambda^{\prime} \boldsymbol{k}}\right)^{2}}$, (a)

(b)

(c)

(d)

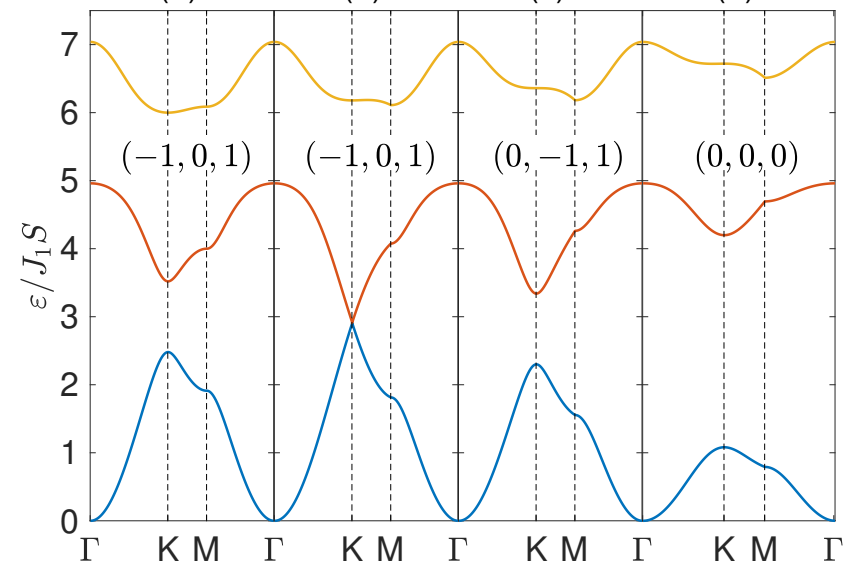

Figure 3. Topological phase transitions of magnon band structures in kagome ferromagnets with selected lattice deformation parameter $\delta=0$ (a), $\delta=0.05$ (b), $\delta=0.1$ (c), $\delta=0.2$ (d). In all panels: the equilibrium value of NN DMI $D=0.3$ and the NNN ferromagnetic coupling $J_{2}=0$. Topological phase is distinguished by its set of Chern numbers $\left(C_{1}, C_{2}, C_{3}\right)$.

where $\psi_{\lambda k}$ and $\varepsilon_{\lambda k}$ are the eigenvectors and eigenvalues of $H_{m}(\boldsymbol{k})$ with band index $\lambda$, respectively. The Chern number of the band $\lambda$ is defined as the integration of the Berry curvature over the Brillouin zone (BZ) shown in Fig. 1(c),

$$
C_{\lambda}=\frac{1}{2 \pi} \int_{B Z} \Omega_{\lambda k}^{z} d^{2} k
$$

The topological phase is specified by the set of Chern numbers $\left(C_{1}, C_{2}, C_{3}\right)$ of the lower, middle and upper magnon bulk bands, as shown in Fig. 3. To investigate the bulk-boundary correspondence of magnons, the winding number of the topologically protected edge modes in band gap $\zeta=1,2$ is defined as $[51,80]$

$$
\nu_{\zeta}=\sum_{\lambda \leq \zeta} C_{\lambda}
$$

The winding number indicates that $\left|\nu_{\zeta}\right|$ pairs of topologically nontrivial edge modes can be found in $\zeta$ th band gap and their propagation direction is given by the sign of $\nu_{\zeta}$.

The existence of nontrivial topological magnon edge modes can be detected by measuring of the transverse thermal Hall response. From the linear response theory, the thermal Hall conductivity connected to the Berry curvature can be expressed as [32]

$$
\kappa_{x y}=-\frac{k_{B}^{2} T}{(2 \pi)^{2} \hbar} \sum_{\lambda=1}^{3} \int_{B Z} c_{2}\left[\rho^{B}\left(\varepsilon_{\lambda k}\right)\right] \Omega_{\lambda k}^{z} d^{2} k,
$$

where $\rho^{B}\left(\varepsilon_{\lambda k}\right)=\left(e^{\varepsilon_{\lambda k} / k_{B} T}-1\right)^{-1}$ is the Bose distribution function. The weighting function is defined as $c_{2}=$ $(1+x) \ln ^{2} \frac{1+x}{x}-\ln ^{2} x-2 \mathrm{Li}_{2}(-x)$ and $\mathrm{Li}_{2}$ is the dilogarithm. 
(a)

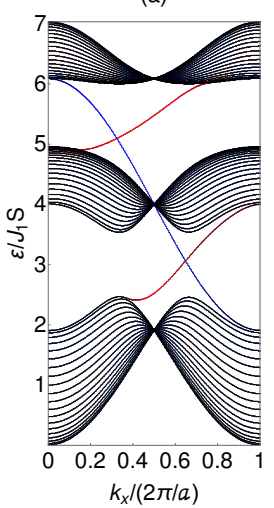

(b)

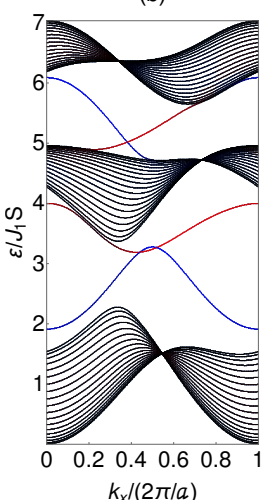

(c)

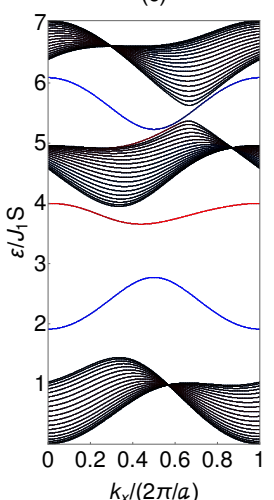

Figure 4. Chiral magnonic edge modes in a kagome lattice. (a) $\delta=0$. (b) $\delta=0.1$. (c) $\delta=0.18$. The black lines are the bulk bands and the blue/red lines are the edge modes. Other parameters are the same as Fig. 3

\section{RESULTS AND DISCUSSION}

To simplify our discussion, we adopt the dimensionless parameters that $J_{1}=S=a=1$ and other quantities are expressed with respect to these parameters. In the following numerical calculations, unless otherwise specified, we set $\eta=2$ and $J_{2}=0$ whereas $D$ and $\delta$ are tunable parameters. We calculate Chern numbers using the algorithm of Fukui et al. with $1000 \times 1000$ points in momentum space [81].

\section{A. Topological phase diagram}

As we mentioned above, the lattice deformation parameter $\delta$ provides a handle to tune topological phase transitions in breathing kagome ferromagnets. The topological phase diagram as a function of $\delta$ and the NN DMI strength $D$ is shown in Fig. 2. Three different topological phases are found with Chern numbers: $(-1,0,1),(0,-1,1)$ and $(0,0,0)$. Since $\delta>0$ and $\delta<0$ are equivalent, which can be directly understood with the mirror symmetry of the two cases to the $x$ axis in Fig. 1(a), we will adopt $\delta>0$ in our following discussion without loss of generality. As the nonzero winding numbers in phase I are $\nu_{1}=\nu_{2}=-1$, two equivalent pairs of topologically protected edge modes will be found in band gap 1 and 2 . In phase II, $\nu_{1}=0$ and $\nu_{2}=-1$ show only one pair of topologically protected edge modes appear in gap 2 . As $\delta$ increased further, the system has two trivial band gap with all three Chern numbers being zero in phase III. In other words, the system can undergo topological phase transitions between two kinds of TMI with chiral nontrivial edge modes (phase I and II) and a trivial magnon insulator without any nontrivial edge modes (phase III) by varying the value of $\delta$ and $D$.

\section{B. Band topology and magnonic edge modes}

Next, we investigate these topological phase transitions though the evolution of the magnon band structures with varying $\delta$ when $D=0.3$. The magnon bands along the $\Gamma-K-M-\Gamma$ line with high-symmetry points in the Brillouin zone are given in Fig. 3. In the equilibrium case $(\delta=0)$, three well separated magnon bands with Chern numbers $(-1,0,1)$ as shown in Fig. 3(a), consistent with previous results [51]. When the lattice deformation parameter reaches the phase transformation line between phase I and II, i.e. $\delta=0.05$, a linear band crossing of the two acoustic magnon branches occurs at the $K$ point in Fig. 3(b). Fig. 3(c) plots the magnon bands at $\delta=0.1$ in phase II. The band gap between the two acoustic magnon branches reopens, while the edge modes transfer from topologically nontrivial modes to trival ones. For comparison, the magnon bands under the influence of a large lattice deformation parameter $\delta=0.2$ are also shown in Fig. 3(d). The system turns out to be a trivial magnon insulator in phase III. The underlying physics can be well understood with the behaviors approaching limiting case $\delta \rightarrow 0.5$, where the positions of three sublattices almost overlap. As the intercell NN exchange interactions are turned off (i.e. $J_{-}=D_{-}=0$ ) in this limit, the system is reduced to an array of isolated trimers on a honeycomb lattice. Since neighboring trimers do not "talk" to each other, the magnon bands degenerate into three flat bands separated by two large trivial gaps.

Furthermore, to better visualize the magnonic edge modes, we have computed the edge modes by solving the eigenvalue problem of a nanoribbon geometry with open boundary conditions in Fig. 4. As shown in Fig. 4(a), two crossed chiral edge modes are clearly found in each band gap, which has been discussed in Ref. [51]. The edge modes are topologically protected and connect the lower and the upper bulk bands. Moreover, the edge modes labeled by blue and red lines have opposite propagation directions (clockwise and anticlockwise rotation). In Fig. 4(b) and Fig. 4(c), the gapped trivial edge modes are observed in trivial band gaps, since the edge modes cannot overcome the increasing width of band gaps due to the increase of lattice deformations [82].

\section{Topological thermal Hall effect}

In this section, we turn to the discussion of topological properties of the magnon THE. In electronic systems, the transverse electrical conductivity is quantized and directly related to the integer values of Chern number, because the Fermi surface can be easily localized in the band gap by doping the system. In contrast, there is no Fermi surface or completely filled bands in magnonic system due to the bosonic statistics. However, the existence of nontrivial topological phases and phase transitions can still be probed by the measurement of the thermal Hall response in inelastic neutron scattering experiments.

In Fig. 5(a), the magnon thermal Hall conductivity $\kappa_{x y}$ is plotted as a function of temperature in three different topolog- 
(a)

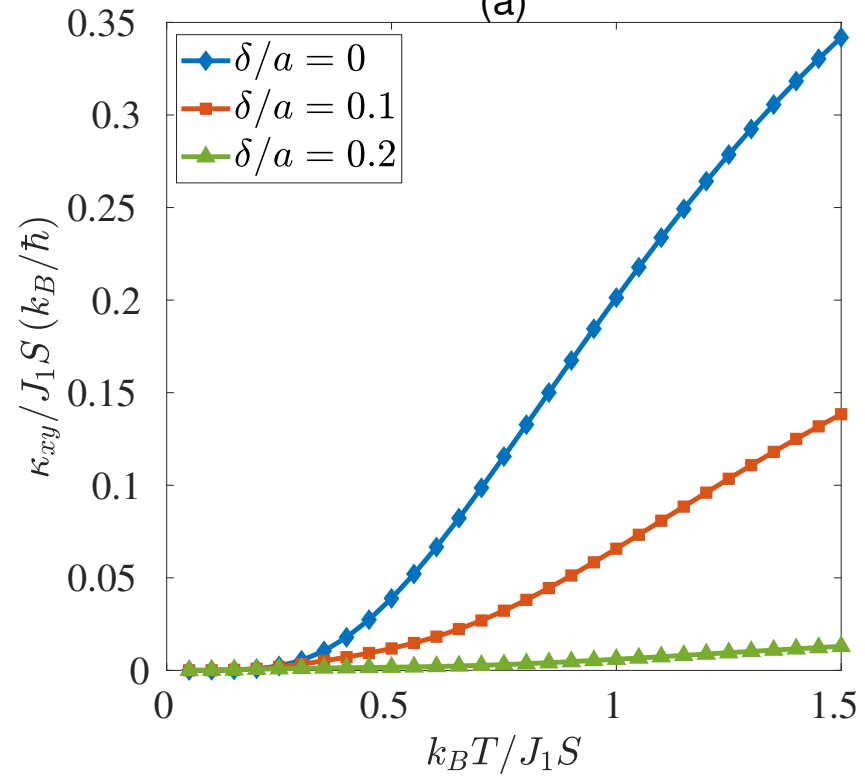

(c)

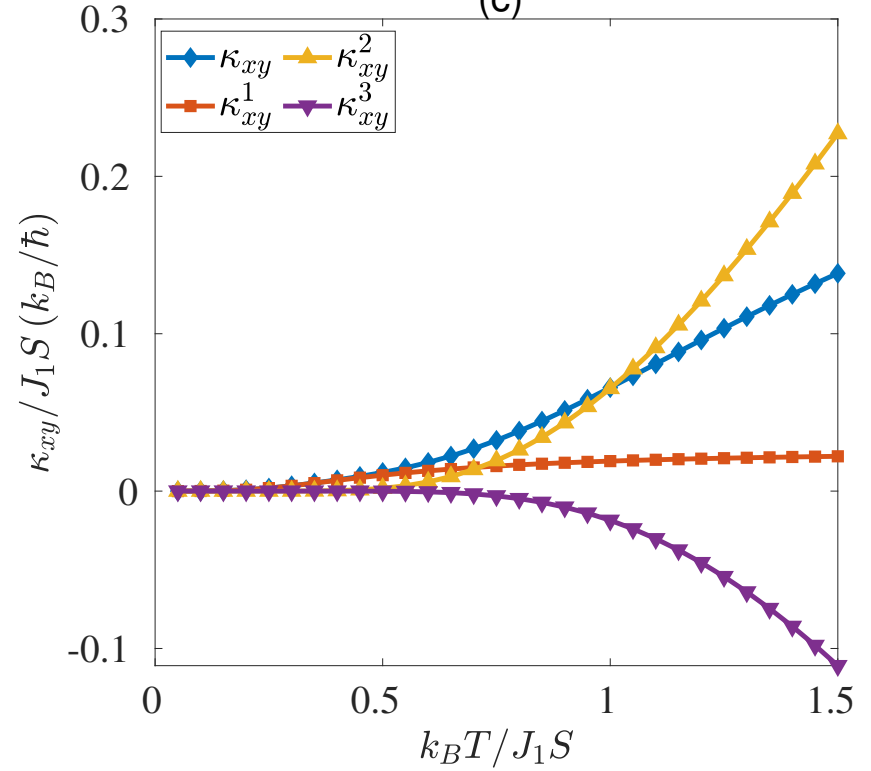

(b)

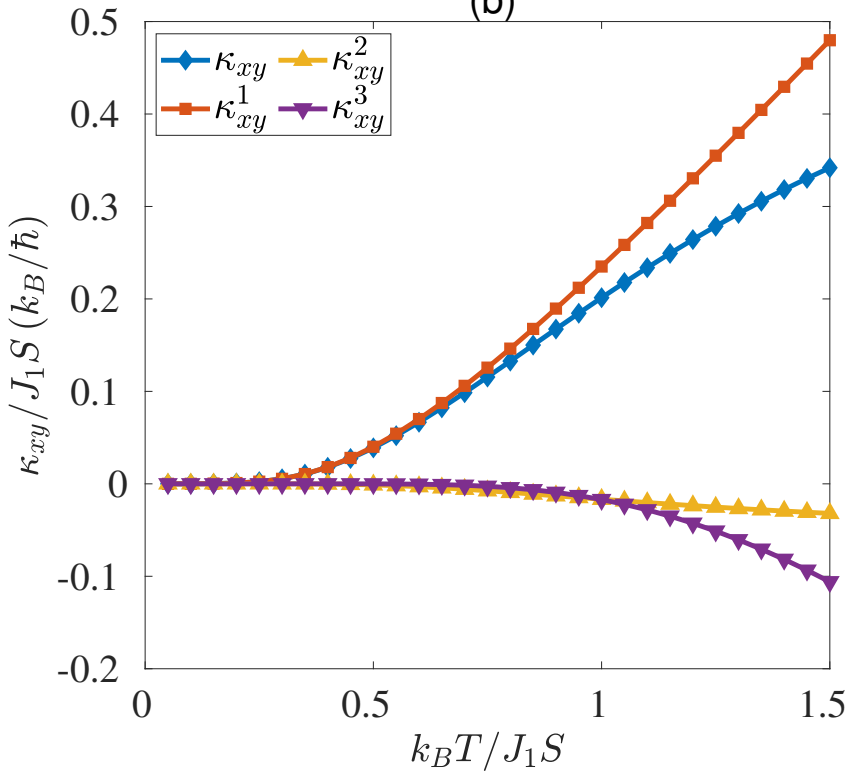

(d)

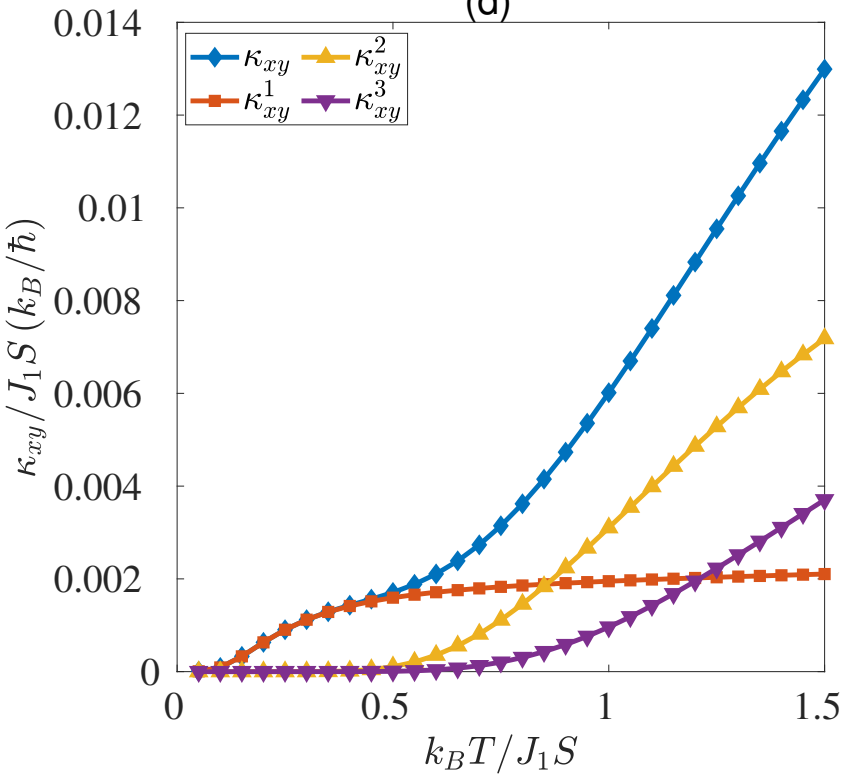

Figure 5. (a) The magnon thermal Hall conductivity as a function of temperature at different lattice deformation parameter in low temperature regime. The magnon thermal Hall conductivity $\left(\kappa_{x y}\right)$ and its band contributions $\left(\kappa_{x y}^{\lambda}\right)$ as a function of temperature when $\delta$ takes the values $(b)$ 0 , (c) 0.1 and (d) 0.2 .

ical phases with different $\delta$ (Fig. 2) in low temperature regime. They all show a monotonically rising behavior. From Eq. (7) and Eq. (9), we find that the Chern number and $\kappa_{x y}$ differ by a constant factor 1 and the weighting function $c_{2}$ in the integrand. To verify the correlation between $\kappa_{x y}$ and $C_{\lambda}$, the temperature dependence of the total conductivity and the contribution from each band $\kappa_{x y}^{\lambda}$ with $\kappa_{x y}=\sum_{\lambda=1}^{3} \kappa_{x y}^{\lambda}$ in different topological phases are plotted in Fig. 5(b)-(d). We find that the significant contributions come from the bands with nonzero Chern numbers $(+1$ or -1$)$. In addition, the band with a zero Chern number also has a nonzero small contribution to the thermal Hall conductivity, and there is a nonzero small thermal Hall conductivity in a trivial magnon insulator [see Fig. 5(d)]. Fig. 5 also shows that the dominant contribution of the thermal Hall conductivity comes from the lowest band at low temperatures, since the lowest band is more occupied due to the weighting effect of $c_{2}$ function with the bosonic nature of magnons. On the contrary, the contributions from upper bands have larger occupation and become significant as the temperature increasing [31].

The magnon thermal Hall conductivity as a function of $\delta$ for four different temperatures is depicted in Fig. 6. Significantly, the NNN ferromagnetic coupling is set to $J_{2}=1$ rather than zero in the previous discussions. Three topological 


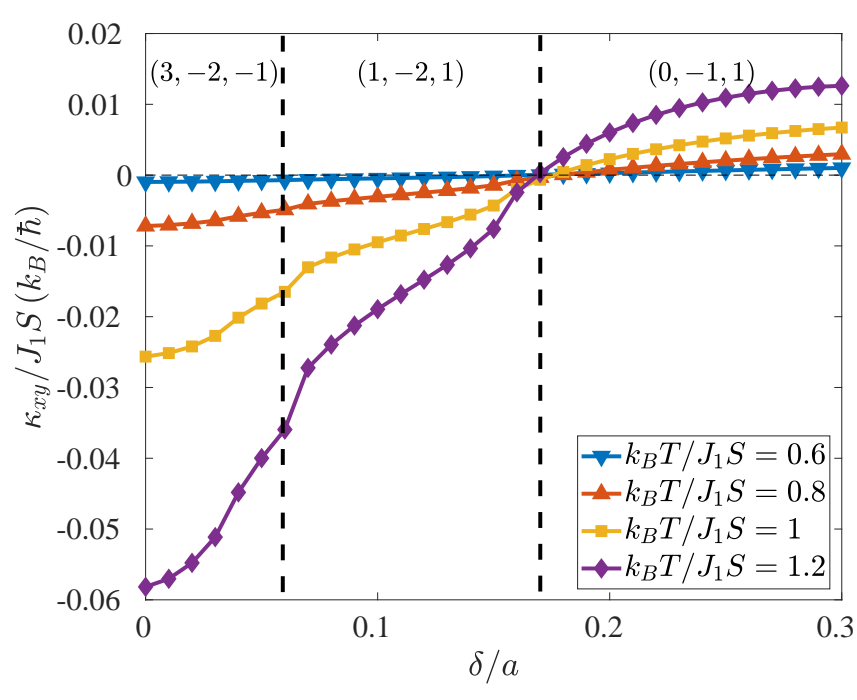

Figure 6. Thermal Hall conductivity versus lattice deformation parameter at different temperatures with the NN DMI $D=0.3$ and NNN ferromagnetic coupling $J_{2}=1$ in low temperature regime. The critical points of phase transition are shown as vertical dashed lines.

phases are found in the range $0 \leq \delta \leq 0.3$, which were separated by the critical points of phase transition represented by the dotted black lines. As the lattice deformation is tuned between the topological phases $(1,-2,1)$ and $(0,-1,1)$, a sign reversal of $\kappa_{x y}$ occurs accompanied by the topological phase transition. However, this sign reversal can be understood explicitly in terms of the propagation direction of chiral edge modes. As mentioned above, the winding number indexs the number and propagation direction of edge modes, which are given as $\nu_{1}=1, \nu_{2}=-1$ in the phase $(1,-2,1)$ and $\nu_{1}=0$, $\nu_{2}=-1$ in the phase $(1,-2,1)$. Although the former has the nontrivial edge modes with opposite propagating direction in the nontrivial gap 1 and gap 2, the edge modes in the lowest gap $\left(\nu_{1}=1\right)$ give the dominant contribution to the thermal Hall conductivity at low temperatures. By contrast, the latter shows nontrivial edge modes only in the upper gap $\left(\nu_{2}=-1\right)$. It also explians why $\kappa_{x y}$ in the phase $(0,-1,1)$ is much lower than in the phase $(1,-2,1)$. Thus the sign of $\kappa_{x y}$ depends on the propagation direction of nontrivial edge modes as well as the occupation probability of the edge magnons, that is, temperatures.

\section{Material consideration}

Before concluding, we shall briefly comment on the candidate materials for an experimental realization of the above topological phase transition in magnonic system. As mentioned above, a skyrmion crystal phase was experimentally observed in the centrosymmetric breathing kagome lattice $\mathrm{Gd}_{3} \mathrm{Ru}_{4} \mathrm{Al}_{12}$ under a field-aligned ferromagnetic phase [74]. This material is insensitive to thermal fluctuations due to the large local spin moments, which makes it an excellent candidate for experiments. The thermal Hall re- sponses are commonly experimentally observed in a number of three-dimensional (3D) ferromagnetic pyrochlore oxides: $\mathrm{Lu}_{2} \mathrm{~V}_{2} \mathrm{O}_{7}, \mathrm{In}_{2} \mathrm{Mn}_{2} \mathrm{O}_{7}$, as well as $\mathrm{Ho}_{2} \mathrm{~V}_{2} \mathrm{O}_{7}$ [17, 25]. Here, instead of considering a real $3 \mathrm{D}$ system, we can realize our ideas by treating it as a stack of non-interacting or weaklyinteracting kagome layers. On the other hand, the thermal Hall effect was also reported in the quasi-2D kagome ferromagnet in $\mathrm{Cu}(1-3, \mathrm{bdc})[26,27]$ or the mineral haydeeite, $\alpha$ $\mathrm{MgCu}_{3}(\mathrm{OD})_{6} \mathrm{Cl}_{2}$ [83], which will also be an interesting platform to investigate the topological phase transition. Since the $\mathrm{NN}$ interactions depend sensitively on the atomic distances, a sizable variation of the NN interactions can be achieved from the lattice distortion by applying external perturbation such as strain or pressure in experiments [84-86]. Therefore, the detailed first-principles calculations and experiments are highly anticipated to investigate the TMI in the future.

\section{CONCLUSION}

We have theoretically demonstrated topological phase transitions due to the lattice deformation in the 2D breathing kagome-lattice ferromagnets with DMI. Having analyzed in detail the band topology of magnons, we show the system can undergo a transition between a TMI with chiral nontrivial edge modes and a trivial magnon insulator without any nontrivial edge modes by tuning the lattice deformation. Moreover, we also calculate the magnon thermal Hall conductivity and expound its relevance to the topological phase and phase transition. We find the reversal of the sign for the thermal Hall conductivity during topological phase transitions. This sign reversal can be applied in the field of thermal micro-sensors in the future, because of its special thermal isolation performance and advantages in technology.

However, the linear spin-wave theory becomes inaccurate at high temperatures, as magnon-magnon interactions and magnon-phonon interactions need to be taken into account [46-49]. Furthermore, tuning the lattice deformation across the wide range is also a practical challenge [53]. We look forward to addressing these issues in our future studies.

\section{ACKNOWLEDGEMENTS}

We would like to acknowledge useful discussions with Xiukun Hua, Jihuan Guan and S. Ghosh. F.Z. was supported by King Abdullah University of Science and Technology (KAUST). A.M. acknowledges support from the Excellence Initiative of Aix-Marseille Université-A*Midex, a French "Investissements d'Avenir" program. H.L. acknowledge the support from Henan University (No. CJ3050A0240050) and National Natural Science Foundation of China (No. 11804078). 


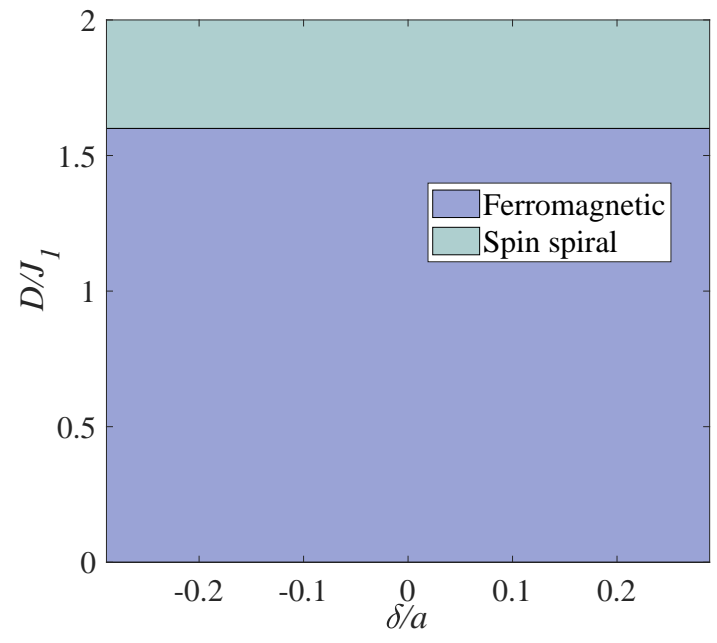

Figure 7. Schematic phase diagram for the ferromagnetic structure and spin spiral.

\section{APPENDIX A: SPIN TEXTURE IN THE GROUND STATE}

Our theoretical work is built on the classical ground state with ferromagnetic ordering. To verify the stability of this ferromagnetic ground state, we estimate the critical value of $D / J_{1}$ by using atomistic spin simulations with the Spirit framework [87], at which there exists a transition from the ferromagnetic order to other magnetic order. In our numerical simulations, we start with a random spin configuration at $t=0$. Then local spin moments quickly evolve into their stable states in a sufficient time after relaxing with Gilbert damping. In Fig. 7 we draw the phase diagram of the magnetic ground state on the $D / J_{1}-\delta / a$ plane, where the other parameters are fixed to the ones we used in the main text: $J_{2}=0$ and $\eta=2$. We see that the critical value $D / J_{1} \sim 1.6$ is entirely unrelated to the lattice deformation parameter $\delta$, consistent with the findings in normal kagome lattices as in Ref. [31]. Above the critical value, the spin spiral is formed instead of the ferromagnetic order. It can be understood in terms of the unaltered total energy under the lattice deformation. These numerical results indicate that the system holds the stable ferromagnetic ground state for a wide range of parameters including the ones we chose in our work.
[1] M. Z. Hasan and C. L. Kane, Rev. Mod. Phys. 82, 3045 (2010).

[2] M. Z. Hasan and J. E. Moore, Annu. Rev. Condens. Matter Phys. 2, 55 (2011).

[3] X.-L. Qi and S.-C. Zhang, Rev. Mod. Phys. 83, 1057 (2011).

[4] B. Yan and S.-C. Zhang, Rep. Prog. Phys. 75, 096501 (2012).

[5] Y. Tokura, K. Yasuda, and A. Tsukazaki, Nat. Rev. Phys. 1, 126 (2019).

[6] W. Witczak-Krempa, G. Chen, Y. B. Kim, and L. Balents, Annu. Rev. Condens. Matter Phys. 5, 57 (2014).

[7] R. Schaffer, E. K.-H. Lee, B.-J. Yang, and Y. B. Kim, Rep. Prog. Phys. 79, 094504 (2016).

[8] M. Hafezi, S. Mittal, J. Fan, A. Migdall, and J. Taylor, Nat. Photon. 7, 1001 (2013).

[9] M. C. Rechtsman, J. M. Zeuner, Y. Plotnik, Y. Lumer, D. Podolsky, F. Dreisow, S. Nolte, M. Segev, and A. Szameit, Nature 496, 196 (2013).

[10] L. Lu, J. D. Joannopoulos, and M. Soljačić, Nat. Photon. 8, 821 (2014).

[11] A. B. Khanikaev and G. Shvets, Nat. Photon. 11, 763 (2017).

[12] T. Ozawa, H. M. Price, A. Amo, N. Goldman, M. Hafezi, L. Lu, M. C. Rechtsman, D. Schuster, J. Simon, O. Zilberberg, et al., Rev. Mod. Phys. 91, 015006 (2019).

[13] L. Zhang, J. Ren, J.-S. Wang, and B. Li, Phys. Rev. Lett. 105, 225901 (2010).

[14] R. Süsstrunk and S. D. Huber, Science 349, 47 (2015).

[15] X. Zhang, M. Xiao, Y. Cheng, M.-H. Lu, and J. Christensen, Commun. Phys. 1, 1 (2018).

[16] H. Katsura, N. Nagaosa, and P. A. Lee, Phys. Rev. Lett. 104, 066403 (2010).

[17] Y. Onose, T. Ideue, H. Katsura, Y. Shiomi, N. Nagaosa, and Y. Tokura, Science 329, 297 (2010).
[18] R. Matsumoto and S. Murakami, Phys. Rev. Lett. 106, 197202 (2011).

[19] R. Shindou, R. Matsumoto, S. Murakami, and J.-i. Ohe, Phys. Rev. B 87, 174427 (2013).

[20] V. Bonbien, F. Zhuo, A. Salimath, O. Ly, A. Abbout, and A. Manchon, J. Phys. D: Appl. Phys. (2021).

[21] X. Wang and X. Wang, J. Appl. Phys. 129, 151101 (2021).

[22] V. Kruglyak, S. Demokritov, and D. Grundler, J. Phys. D: Appl. Phys. 43, 264001 (2010).

[23] A. Serga, A. Chumak, and B. Hillebrands, J. Phys. D: Appl. Phys. 43, 264002 (2010).

[24] A. V. Chumak, V. I. Vasyuchka, A. A. Serga, and B. Hillebrands, Nat. Phys. 11, 453 (2015).

[25] T. Ideue, Y. Onose, H. Katsura, Y. Shiomi, S. Ishiwata, N. Nagaosa, and Y. Tokura, Phys. Rev. B 85, 134411 (2012).

[26] M. Hirschberger, R. Chisnell, Y. S. Lee, and N. P. Ong, Phys. Rev. Lett. 115, 106603 (2015).

[27] R. Chisnell, J. S. Helton, D. E. Freedman, D. K. Singh, R. I. Bewley, D. G. Nocera, and Y. S. Lee, Phys. Rev. Lett. 115, 147201 (2015).

[28] A. Manchon, H. C. Koo, J. Nitta, S. Frolov, and R. Duine, Nat. Mater. 14, 871 (2015).

[29] A. Belabbes, G. Bihlmayer, F. Bechstedt, S. Blügel, and A. Manchon, Phys. Rev. Lett. 117, 247202 (2016).

[30] A. Manchon, J. Železný, I. M. Miron, T. Jungwirth, J. Sinova, A. Thiaville, K. Garello, and P. Gambardella, Rev. Mod. Phys. 91, 035004 (2019).

[31] A. Mook, J. Henk, and I. Mertig, Phys. Rev. B 89, 134409 (2014).

[32] R. Matsumoto, R. Shindou, and S. Murakami, Phys. Rev. B 89, 054420 (2014).

[33] S. A. Owerre, Phys. Rev. B 94, 094405 (2016). 
[34] S. S. Pershoguba, S. Banerjee, J. C. Lashley, J. Park, H. Ågren, G. Aeppli, and A. V. Balatsky, Phys. Rev. X 8, 011010 (2018).

[35] K.-S. Kim, K. H. Lee, S. B. Chung, and J.-G. Park, Phys. Rev. B 100, 064412 (2019).

[36] S. A. Owerre, Phys. Rev. B 95, 014422 (2017).

[37] P. Laurell and G. A. Fiete, Phys. Rev. B 98, 094419 (2018).

[38] Y. Lu, X. Guo, V. Koval, and C. Jia, Phys. Rev. B 99, 054409 (2019).

[39] A. Mook, J. Henk, and I. Mertig, Phys. Rev. B 99, 014427 (2019).

[40] B. Göbel, A. Mook, J. Henk, and I. Mertig, Phys. Rev. B 95, 094413 (2017).

[41] S. A. Díaz, J. Klinovaja, and D. Loss, Phys. Rev. Lett. 122 , 187203 (2019).

[42] S. A. Díaz, T. Hirosawa, J. Klinovaja, and D. Loss, Phys. Rev. Research 2, 013231 (2020).

[43] B. A. Bernevig, T. L. Hughes, and S.-C. Zhang, Science 314, 1757 (2006).

[44] S.-Y. Xu, Y. Xia, L. Wray, S. Jia, F. Meier, J. Dil, J. Osterwalder, B. Slomski, A. Bansil, H. Lin, et al., Science 332, 560 (2011).

[45] J. Romhányi, K. Penc, and R. Ganesh, Nat. Commun. 6, 1 (2015).

[46] X. Zhang, Y. Zhang, S. Okamoto, and D. Xiao, Phys. Rev. Lett. 123, 167202 (2019).

[47] E. Thingstad, A. Kamra, A. Brataas, and A. Sudbø, Phys. Rev. Lett. 122, 107201 (2019).

[48] S. Zhang, G. Go, K.-J. Lee, and S. K. Kim, Phys. Rev. Lett. 124, 147204 (2020).

[49] Y.-S. Lu, J.-L. Li, and C.-T. Wu, arXiv 2104, 00266 (2021).

[50] S. K. Kim, H. Ochoa, R. Zarzuela, and Y. Tserkovnyak, Phys. Rev. Lett. 117, 227201 (2016).

[51] A. Mook, J. Henk, and I. Mertig, Phys. Rev. B 90, 024412 (2014).

[52] C. Moulsdale, P. A. Pantaleón, R. Carrillo-Bastos, and Y. Xian, Phys. Rev. B 99, 214424 (2019).

[53] Y.-H. Li and R. Cheng, Phys. Rev. B 103, 014407 (2021).

[54] S. Capponi, A. Läuchli, and M. Mambrini, Phys. Rev. B 70, 104424 (2004).

[55] F. H. Aidoudi, D. W. Aldous, R. J. Goff, A. M. Slawin, J. P. Attfield, R. E. Morris, and P. Lightfoot, Nat. Chem. 3, 801 (2011).

[56] L. Clark, J. C. Orain, F. Bert, M. A. De Vries, F. H. Aidoudi, R. E. Morris, P. Lightfoot, J. S. Lord, M. T. F. Telling, P. Bonville, J. P. Attfield, P. Mendels, and A. Harrison, Phys. Rev. Lett. 110, 207208 (2013).

[57] J.-C. Orain, B. Bernu, P. Mendels, L. Clark, F. H. Aidoudi, P. Lightfoot, R. E. Morris, and F. Bert, Phys. Rev. Lett. 118, 237203 (2017).

[58] R. Schaffer, Y. Huh, K. Hwang, and Y. B. Kim, Phys. Rev. B 95, 054410 (2017).

[59] C. Repellin, Y.-C. He, and F. Pollmann, Phys. Rev. B 96, 205124 (2017).

[60] A. Akbari-Sharbaf, R. Sinclair, A. Verrier, D. Ziat, H. D. Zhou, X. F. Sun, and J. A. Quilliam, Phys. Rev. Lett. 120, 227201 (2018).

[61] M. Ezawa, Phys. Rev. Lett. 120, 026801 (2018).

[62] A. Bolens and N. Nagaosa, Phys. Rev. B 99, 165141 (2019).

[63] S. Kempkes, M. Slot, J. van Den Broeke, P. Capiod, W. Benalcazar, D. Vanmaekelbergh, D. Bercioux, I. Swart, and C. M. Smith, Nat. Mater. 18, 1292 (2019).

[64] K. Kudo, T. Yoshida, and Y. Hatsugai, Phys. Rev. Lett. 123, 196402 (2019).

[65] A. El Hassan, F. K. Kunst, A. Moritz, G. Andler, E. J. Bergholtz, and M. Bourennane, Nat. Photon. 13, 697 (2019).
[66] M. Li, D. Zhirihin, M. Gorlach, X. Ni, D. Filonov, A. Slobozhanyuk, A. Alù, and A. B. Khanikaev, Nat. Photon. 14, 89 (2020).

[67] X. Ni, M. Weiner, A. Alu, and A. B. Khanikaev, Nat. Mater. 18, 113 (2019)

[68] H. Xue, Y. Yang, F. Gao, Y. Chong, and B. Zhang, Nat. Mater. 18, 108 (2019).

[69] M. Ezawa, Phys. Rev. B 98, 201402 (2018).

[70] H. Yang, Z.-X. Li, Y. Liu, Y. Cao, and P. Yan, Phys. Rev. Research 2, 022028 (2020).

[71] L. Song, H. Yang, Y. Cao, and P. Yan, Nano Letters 20, 7566 (2020).

[72] Z. Li, Y. Cao, P. Yan, and X. Wang, npj Comput. Mater. 5, 1 (2019).

[73] H. Wakao, T. Yoshida, H. Araki, T. Mizoguchi, and Y. Hatsugai, Phys. Rev. B 101, 094107 (2020).

[74] M. Hirschberger, T. Nakajima, S. Gao, L. Peng, A. Kikkawa, T. Kurumaji, M. Kriener, Y. Yamasaki, H. Sagayama, H. Nakao, et al., Nat. Commun. 10, 1 (2019).

[75] K. Essafi, L. Jaubert, and M. Udagawa, J. Phys.: Condens. Matter 29, 315802 (2017).

[76] A. Sil and A. K. Ghosh, J. Phys.: Condens. Matter 32, 205601 (2020).

[77] M. A. Vozmediano, M. Katsnelson, and F. Guinea, Phys. Rep. 496, 109 (2010).

[78] Y. Ferreiros and M. A. Vozmediano, Phys. Rev. B 97, 054404 (2018).

[79] T. Holstein and H. Primakoff, Phys. Rev. 58, 1098 (1940).

[80] Y. Hatsugai, Phys. Rev. Lett. 71, 3697 (1993).

[81] T. Fukui, Y. Hatsugai, and H. Suzuki, J. Phys. Soc. Jpn. 74, 1674 (2005).

[82] R. Hidalgo-Sacoto, R. I. Gonzalez, E. E. Vogel, S. Allende, J. D. Mella, C. Cardenas, R. E. Troncoso, and F. Munoz, Phys. Rev. B 101, 205425 (2020).

[83] D. Boldrin, B. Fåk, M. Enderle, S. Bieri, J. Ollivier, S. Rols, P. Manuel, and A. S. Wills, Phys. Rev. B 91, 220408 (2015).

[84] F. Guinea, M. Katsnelson, and A. Geim, Nat. Phys. 6, 30 (2010).

[85] M. Bahramy, B.-J. Yang, R. Arita, and N. Nagaosa, Nat. Commun. 3, 1 (2012).

[86] J. Ruan, S.-K. Jian, H. Yao, H. Zhang, S.-C. Zhang, and D. Xing, Nat. Commun. 7, 1 (2016).

[87] G. P. Müller, M. Hoffmann, C. Dißelkamp, D. Schürhoff, S. Mavros, M. Sallermann, N. S. Kiselev, H. Jónsson, and S. Blügel, Phys. Rev. B 99, 224414 (2019). 